\title{
Combining 3D Interpolation, Regression, and Body Features to build 3D Human Data for Garment: An Application to Building 3D Vietnamese Female Data Model
}

\author{
Tran Thi Minh Kieu ${ }^{1}$ \\ School of Textile - Leather and Fashion, Hanoi University \\ of Science and Technology, Hanoi, Vietnam
}

Nguyen Tung Mau ${ }^{2}$

School of Textile - Leather and Fashion

Hanoi University of Science and Technology

Industrial University of Hochiminh City

Hochiminh City, Vietnam

\author{
Le $\mathrm{Van}^{3}$ \\ Information Science Faculty \\ Sai Gon University, Hochiminh City, Vietnam
}

\author{
Pham The Bao ${ }^{4}$ \\ Information Science Faculty \\ Sai Gon University \\ Hochiminh City \\ Vietnam
}

\begin{abstract}
Modeling 3D human body is an advanced technique used in human motion analysis and garment industry. In this paper, we propose a method for forming deformation functions so that we can rebuild the 3D human body given anthropometric measurements. The advanced idea in our approach is that we split the 3D body into small parts. In that way, we can specialize different set of parameters needed to interpolate for each section. With an interpolation approach, we build a 3D human body for 593 female bodies with the corresponding body shape but require fewer input measurements than 3D laser scans.
\end{abstract}

Keywords-Anthropometry; $3 D$ scanning; human body modeling; interpolation; parametric modeling

\section{INTRODUCTION}

With the rapid increase in 3D technology, 3D human models have more practical applications than ever. In the field of animation, 3D virtual characters used in films or videos are much similar to a real human. Hologram call will be the next step for our telecommunications industry and especially for modern life. When people need more time for their family and work, virtual try-on technology or custom suit online will be cut off the time for shopping. However, full body 3D scanners nowadays are still unaffordable for individual [4]. Besides, scanning systems usually are complicated to set up, and there is rarely a friendly manual.

Those reasons have fueled us to propose a more convenient method for non-expert users, who want a 3D body ourselves without the 3D scanner. With the slightly greater number of key points compared to those used in traditional manners, but much smaller than those used in 3D body scanner, we can estimate 3D human models simply by using a personal computer. To achieve this work, we choose $\mathrm{n}$ basedslices to present the whole human body, then fine-tune sliceby-slice to adapt to any particular human body shapes. Finally, meshes are used to connect the entire body.

We rely on tailor's (circumference) measurements at specific places on the human body to construct essential

This research was supported by the Hanoi University of Science and Technology (HUST) under project number T2018-PC-048. shapes. We build data points with their respective shapes and perimeters to get important slices. From these data slices, we use interpolation to estimate intermediate slices that form the entire 3D body of a human. The dataset used in our experiment contains 593 3D women models which are saved under "obj" format; each model has a set of measurements that is the input of our method. This dataset is from the garment research group of Hanoi University of Technology. We use relative error to compute the error level of the proposed approach; the average square error is 11.02621202 .

\section{RELATED WORKS}

Many techniques have been developing in the field of 3D modeling. The following approaches are vital to the 3D garment design [2].

- Parametric learning: this method creates a human template and then combines with semantic features from an individual to produce a new human model [5].

Wang et al. [1] proposed a method to create a parametric model based on the vital body sizes and Spline interpolation. To satisfy the garment or animation's requirement, the author presented two modules: the skeleton fitting and independent cross sections for each body part. After having a surrogate model, the users can manipulate the key dimensions of the surrogate model to generate their desired 3D model. Cordier et al. [3] developed an Online Clothing Store as a web application. The application requested eight primary measurements of the human body as an input to a generic model. Deforming existing model in a database will be easier to obtain a new one.

- Statistics-Based Synthesis Model: This method generates a human body based on the investigation of the body shape distribution. However, this method does not have a true technique to build a new individual model from the input constraints $[1,9]$. 


\section{METHODOLOGY}

\section{A. Overview}

In this paper, we use the Hermite Curve Interpolation method to interpolate our model. Hermite curves are widely used in computer graphics and geometric models to obtain motion curves or orbits that pass through certain points of the plane or three-dimensional space. In these applications, each coordinate of the plane or space is interpolated using parameters which represent the tension of the curve. We use this method because the curve properties on the human body [9].

In Hermite's method, the third is determined by the first and last points with two tilt angles at those two points. Hermite curves are often used to interpolate data at given values $x_{1}, x_{2}, \ldots, x_{n}$ to achieve a "smoth" continuous function. The data includes the value of the desired function and the derivative at each $x_{k}$. The Hermite formula is applied to each interval $\left(x_{k}, x_{k+1}\right)$ separately. Polynomial interpolation of the Hermite curve is given in formula (1), and the example is shown in figure 1 .

$p(t)=m_{1} h_{3}(t)+p_{1} h_{1}(t)+p_{2} h_{2}(t)+m_{2} h_{4}(t)$

with $m_{1}, m_{2}, p_{1}, p_{2}$ are tangential values and $h_{1}(t), h_{2}(t), h_{3}(t), h_{4}(t)$ are the basic Hermit functions corresponding to

$h_{1}(t)=2 t^{3}-3 t^{2}+1$

$h_{2}(t)=-2 t^{3}+3 t^{2}$

$h_{3}(t)=t^{3}-2 t^{2}+t$

$h_{3}(t)=t^{3}-2 t^{2}+t$

The Hermite curve with tension parameters is formulated as (2).

$p(t)=\left[\begin{array}{llll}t^{3} & t^{2} & t & 1\end{array}\right]\left[\begin{array}{cccc}2 & -2 & 1 & 1 \\ -3 & 3 & -2 & -1 \\ 0 & 0 & 1 & 0 \\ 1 & 0 & 0 & 0\end{array}\right]\left[\begin{array}{c}p_{1} \\ p_{2} \\ m_{1} \\ m_{2}\end{array}\right]$

with $m_{1}=s\left(p_{2}-p_{0}\right)$ and $m_{2}=s\left(p_{3}-p_{1}\right)$

Then we have (3),

$p(t)=\left[\begin{array}{llll}t^{3} & t^{2} & t & 1\end{array}\right]$

$\left[\begin{array}{cccc}2 & -2 & 1 & 1 \\ -3 & 3 & -2 & -1 \\ 0 & 0 & 1 & 0 \\ 1 & 0 & 0 & 0\end{array}\right]\left[\begin{array}{cccc}0 & 1 & 0 & 0 \\ 0 & 0 & 1 & 0 \\ -s & 0 & s & 0 \\ 0 & -s & 0 & s\end{array}\right]\left[\begin{array}{l}p_{0} \\ p_{1} \\ p_{2} \\ p_{3}\end{array}\right]$

We generalize to (4).

$p(t)=\left[\begin{array}{llll}t^{3} & t^{2} & t & 1\end{array}\right]\left[\begin{array}{cccc}-s & 2-s & s-2 & s \\ 2 s & s-3 & 3-2 s & -s \\ -s & 0 & s & 0 \\ 0 & 1 & 0 & 0\end{array}\right]\left[\begin{array}{c}p_{k-1} \\ p_{k} \\ p_{k+1} \\ p_{k+2}\end{array}\right]$

\section{B. Building Model}

1) General model: Diagram 1 shows our proposed framework to generate a 3D human model given a set of hand measurements.

2) Building a set of measurements for the model: The Standard measurements needed to make clothes are listed in Table 1 and 2; these are fundamental measures used in the field of costume design $[3,6]$.

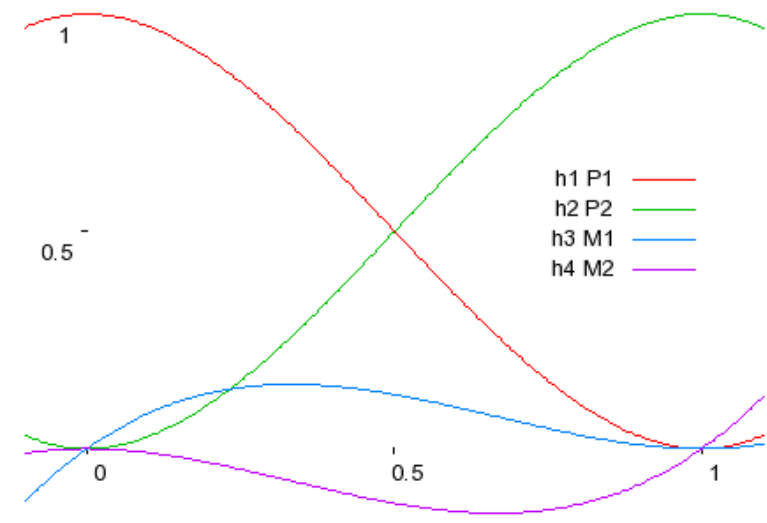

Fig. 1. The basic Hermit Functions.

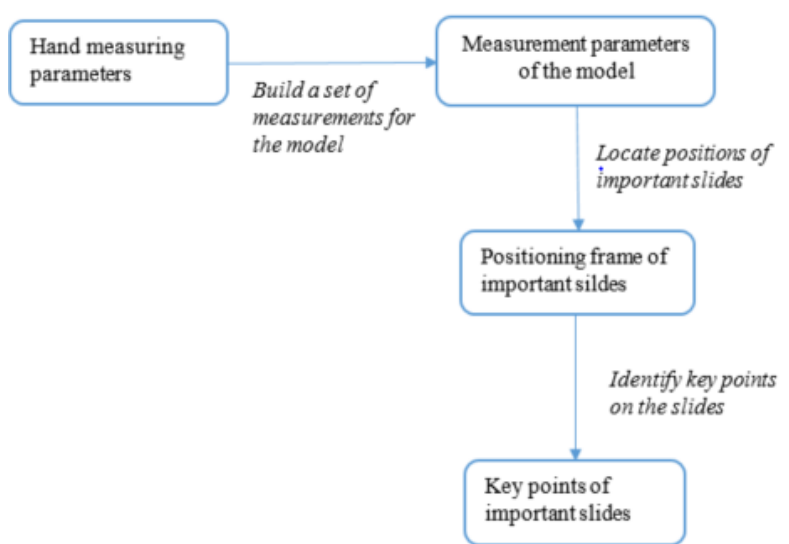

Adjust key points with corresponding hand measurements

The key points after being ajusted

Computing principal slides from key points by interpolation

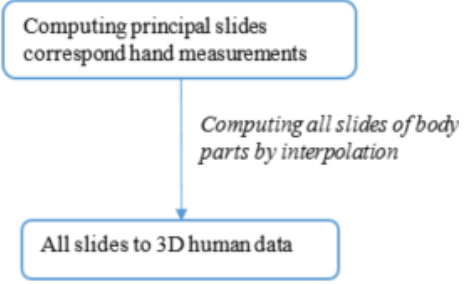

Diagram. 1. General Model. 
TABLE. I. BASIC MEASUREMENTS FOR SEWING Clothes

\begin{tabular}{|l|l|l|}
\hline No & Measure names & Measurement methods \\
\hline 1 & Shoulder & $\begin{array}{l}\text { Measure the distance from the tip of the } \\
\text { shoulder to the side of the shoulder and the } \\
\text { other shoulder }\end{array}$ \\
\hline 2 & Shirt length & $\begin{array}{l}\text { Measure the distance from the 7th vertebrae } \\
\text { of the spine to the level of the perineum }\end{array}$ \\
\hline 3 & Short sleeve length & $\begin{array}{l}\text { Measure the distance from the shoulder to } \\
\text { the elbow }\end{array}$ \\
\hline 4 & Long sleeve length & $\begin{array}{l}\text { Measure the distance from the shoulder to } \\
\text { the wrist }\end{array}$ \\
\hline 5 & Necklace & $\begin{array}{l}\text { Use a tape to measure the circumference } \\
\text { around the neck }\end{array}$ \\
\hline 6 & Chest circumference & $\begin{array}{l}\text { Use a tape to measure the circumference of } \\
\text { the chest }\end{array}$ \\
\hline 7 & Waist circumference & $\begin{array}{l}\text { Use a measuring tape to measure the } \\
\text { circumference of the belly circumference } \\
\text { above the navel } 2 \mathrm{~cm}\end{array}$ \\
\hline
\end{tabular}

TABLE. II. BASIC MEASUREMENT For SEWING PANT

\begin{tabular}{|l|l|l|}
\hline No & Measure names & Measurement methods \\
\hline 1 & Long bottom & $\begin{array}{l}\text { Measure the distance from the waistline to } \\
\text { the bottom of the perineum }\end{array}$ \\
\hline 2 & Short pants & $\begin{array}{l}\text { Measure the vertical distance from the } \\
\text { perineum to the level of the knee }\end{array}$ \\
\hline 3 & Long pants & $\begin{array}{l}\text { Measure the distance from the perineum to } \\
\text { the ground }\end{array}$ \\
\hline 5 & Waist circumference & $\begin{array}{l}\text { Use a measuring tape to measure the } \\
\text { circumference of the belly circumference } \\
\text { above the navel 2 cm }\end{array}$ \\
\hline 6 & Middle thigh & $\begin{array}{l}\text { Use the tape to measure the circumference of } \\
\text { the butt ring at the most protruding level of } \\
\text { the large transfer tab }\end{array}$ \\
\hline 7 & Calf ring & $\begin{array}{l}\text { Use a measuring tape on the circumference } \\
\text { of the thigh, not tighten the ruler, measure } \\
\text { the middle half between the hip and the knee }\end{array}$ \\
\hline 8 & Leg ring & $\begin{array}{l}\text { Use the horizontal measuring tape to circle } \\
\text { the calf of the most enlarged calf }\end{array}$ \\
\hline
\end{tabular}

From the above basic measurements, we build a set of measuring parameters to create a suitable $3 \mathrm{D}$ model in the field of costume design. This set of parameters includes 9 measurements of the circumference of the body, and 7 measurements of body length/width are shown in Table $3[1$, 7].

3) Building a model to locate the position of pricipal slices: The principal slices are at positions that represent the body measurements of the 3D model; we locate those slices to interact with the hand measurements. From measurements of the length of the set of parameters, we can locate the primary positions of the body parts such as calf, thigh, buttocks, abdomen, chest, and neck. It is also easy to locate the slices to be used to change the measurement in each region. A set of frames being interacted with is constructed as shown in Figure 2 , and the meanings are explained in Table 4. We build algorithm 1 to locate the position of principal slices.
TABLE. III. MEASUREMENT PARAMETERS FOR 3D MOdELS ARE BUILT

\begin{tabular}{|l|l|l|}
\hline No & Measure names & \multirow{3}{*}{ Measurement methods } \\
\hline 1 & Necklace & \\
\hline 2 & Chest ring & \multirow{3}{*}{ circumference } \\
\hline 3 & Waist circumference & \\
\hline 4 & Buttocks & \\
\hline 5 & Middle thigh & \\
\hline 6 & Calf circumference & \\
\hline 7 & Leg circumference & \\
\hline 8 & Round the upper arm & \multirow{3}{*}{ length/width } \\
\hline 9 & Wrist cuff & \\
\hline 10 & Length from nape to belly & \\
\hline 11 & Length from belly to perineum & \\
\hline 12 & Thigh length & \\
\hline 13 & Calf length & \\
\hline 14 & Upper arm length & \\
\hline 15 & Lower arm length & \\
\hline 16 & Shoulder width & \\
\hline
\end{tabular}

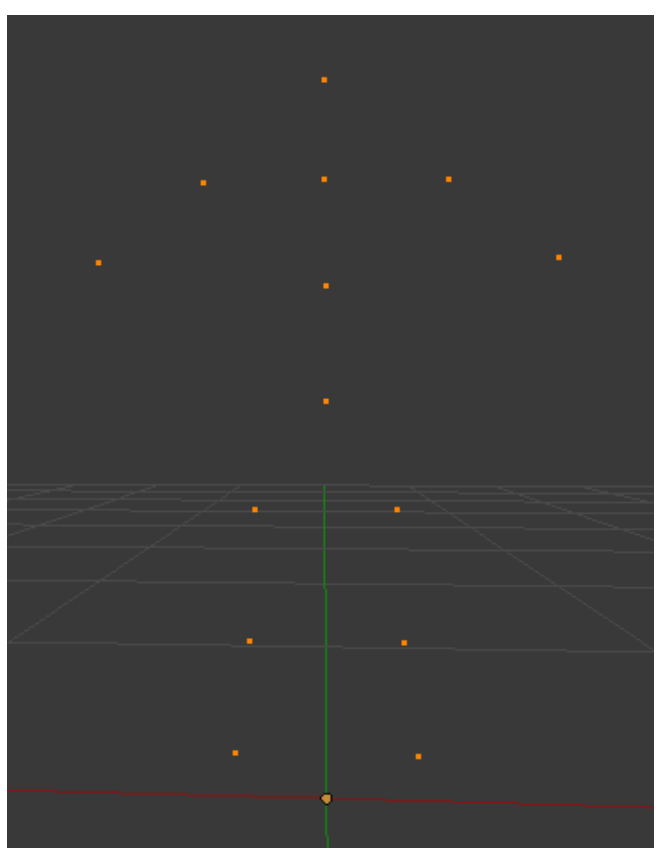

Fig. 2. The Positioning Frame of Principal Slices will be Interacted.

TABLE. IV. MEANING OF POSITIONS

\begin{tabular}{|l|l|}
\hline Position & Meaning \\
\hline$(1)$ & Location of the cross section \\
\hline$(2)$ & Chest circumference position \\
\hline$(3)$ & The position of the abdomen cross section \\
\hline$(4)$ & The position of the buttocks section \\
\hline$(5)$ & The position of the middle thigh section \\
\hline$(6)$ & Face position cuts the calf ring \\
\hline$(7)$ & The position of the cut leg face \\
\hline$(8)$ & The cross-sectional position of the upper arm \\
\hline$(9)$ & The cross-sectional location of the wrist \\
\hline
\end{tabular}


Algorithm 1 Locating the position of principal slices

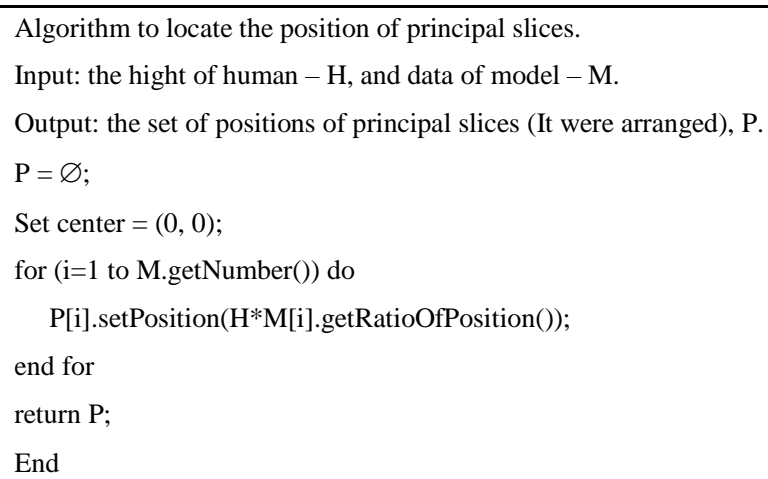

4) Building a model that identifies the key points of each slice: Each human body can be modeled simply by a set of slices, and each slice will contain points to form a curve. To create a controllable human body model, we need to identify some key points on each slice and use these points to create a curve. To find the key points of each slice, we improved the technique which was introduced by Shuxia Wang [1] as follows:

- Kind 1: all slices on the body parts (except the body) have a nearly circular structure, so we will choose 4 extreme points to make the key points of the section, Figure 3 and 4.

- Kind 2: the slices on the body have the complex structure; we observe the symmetry and make statistics to find the 12 key points representing the slice as shown, Figures 5 and 6.

we designed algorithm 2 to solve this problem.

Algorithm 2 Determine the kind for each principal slice.

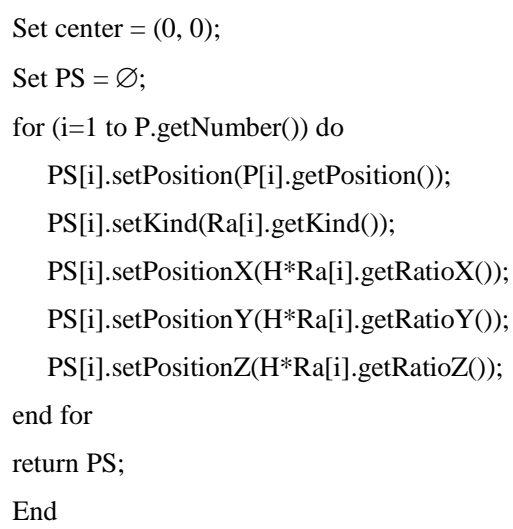

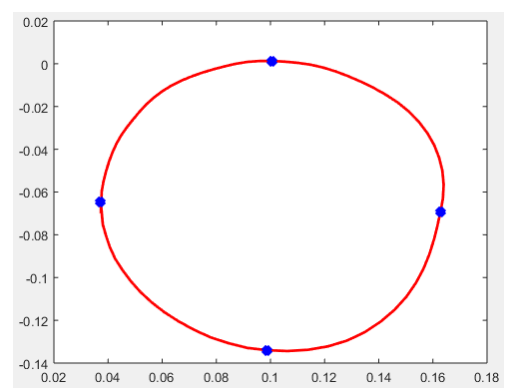

Fig. 3. The Key Points of the Thing Slice.

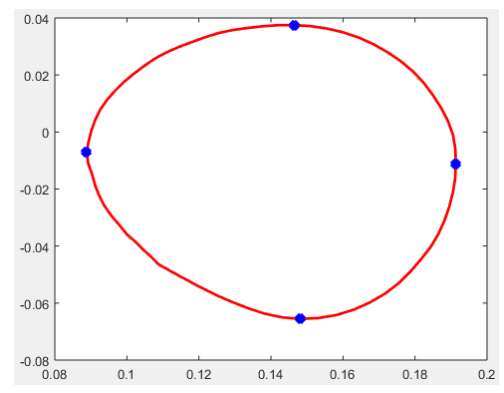

Fig. 4. The Key Points of the Calf Slice.

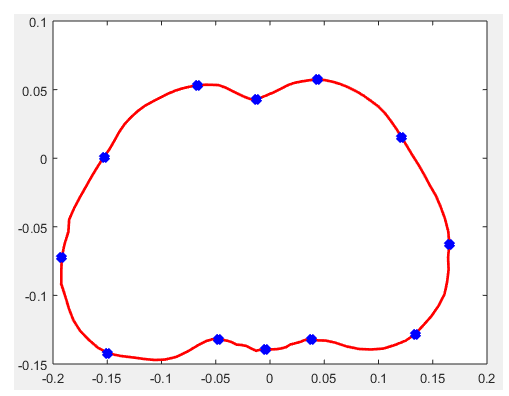

Fig. 5. The Key Points of the Buttocks Slice.

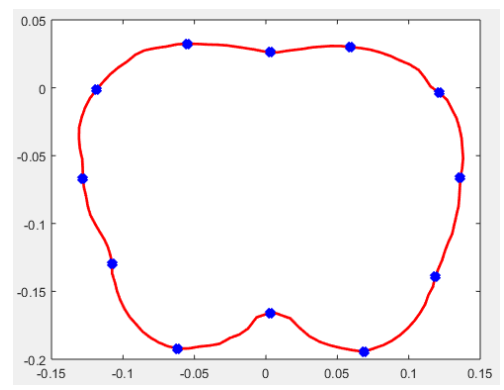

Fig. 6. The Key Points of the Chest Slice.

5) Building a model to adjust the key points on the slice corresponding to hand measurements: Once having the key points which represent each slice, we adjust those points to fit the slice shape with the use of approximate circumferences based on hand measurements, algorithm 3 . We conduct the following steps:

- Step 1: Determine the center, and compute the ratio of the large radius, and small radius of the current slice of the model, Figure 7.

- Step 2: Find the large radius of the current slice of the model $($ Radius $=$ Perimeter $/(2 * \pi))$, Figure 8 . 
- Step 3: Compute the large radius of the real slice based on the hand measurement parameters (Radius = Measure / $(2 * \pi))$, Figure 9 .

- Step 4: Adjust the points corresponding to the large radius of the new slice, Figure 10.

- Step 5: Adjust the point on the small radius in proportion to the large radius of the current slice, Figure 11. slice.

Algorithm 3 Adjusting the key points of one principal
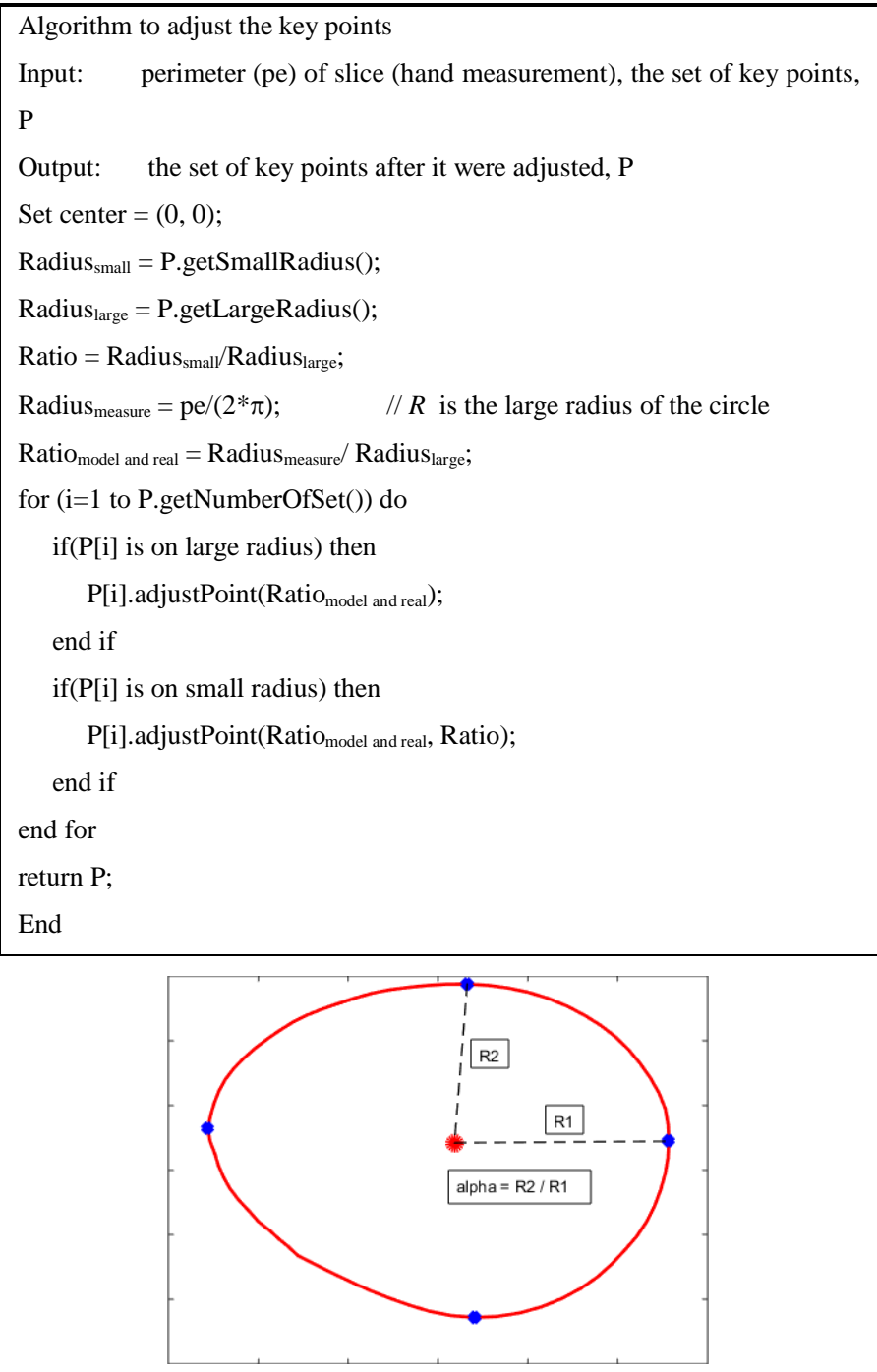

Fig. 7. Step 1 of the Solution.

6) Building the principal slice from key points by interpolation: After having key points on each principal slice, we interpolate more points to create the curve corresponding to the slices. We use Hermite Curve Interpolation method to perform interpolation because of three following reasons:

- This curve can pass through key points of the slice smoothly and without corners.
- This curve does not need to include tangential values at points. Its slope at each point will be computed from the coordinates of the two adjacent points.

- This curve can be controlled by a tension parameter, with different tension parameter values creating different curves.

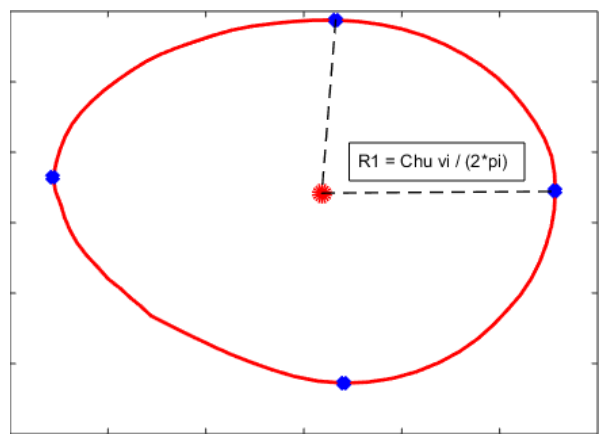

Fig. 8. Step 2 of the Solution.

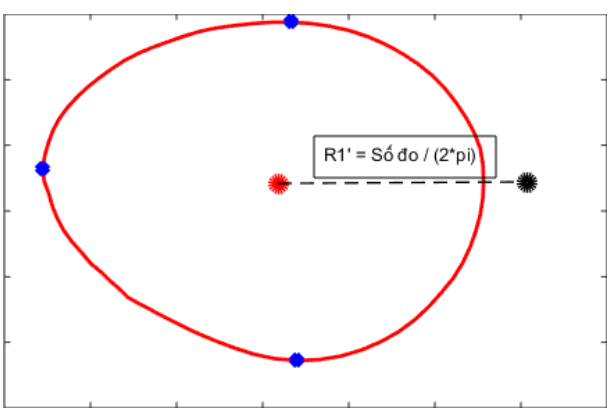

Fig. 9. Step 3 of the Solution.

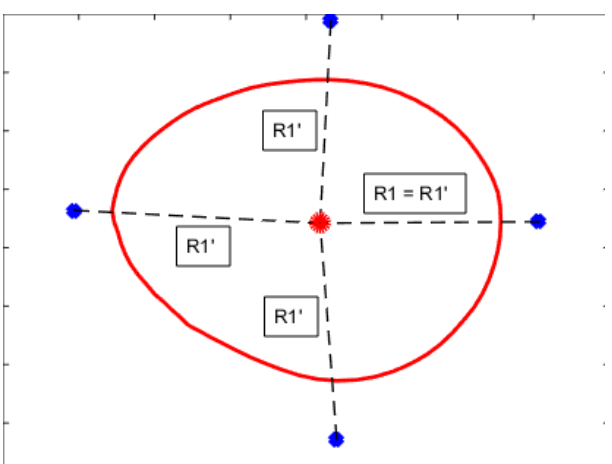

Fig. 10. Step 4 of the Solution.

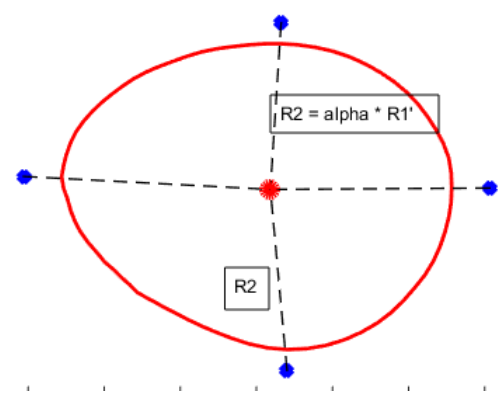

Fig. 11. Step 5 of the Solution. 
Based on the experimental results as well as the results of Shuxia Wang [1], the tension parameter of -0.4 is suitable for the curve of the human body profile. The appropriateness is shown in the figures depicted below with the red "o" points as the raw data points on the slice scanned from the 3D Scanner, the red curve with the tension parameter of 0.8 , the blue curve has a tension parameter of -1.6 and a black curve with a tension parameter of -0.4 , Figure 12, 13. We use Algorithm 4 to generate data points for the principal slices for the body, Figure 14.

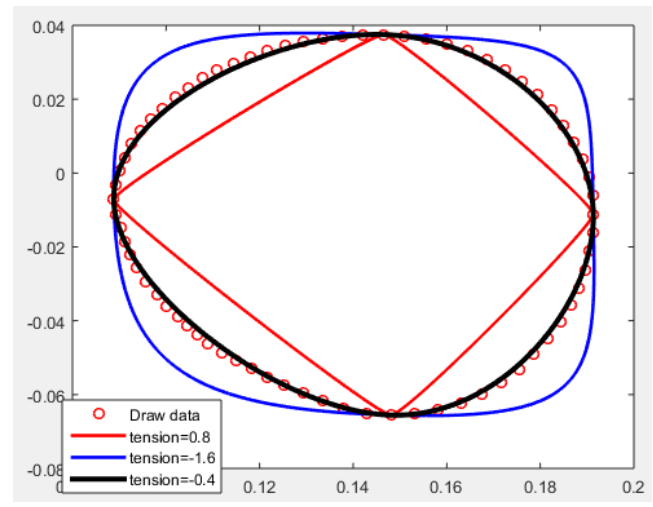

Fig. 12. Interpolation Curvers from 4 Key Points with different Tension Parameters.

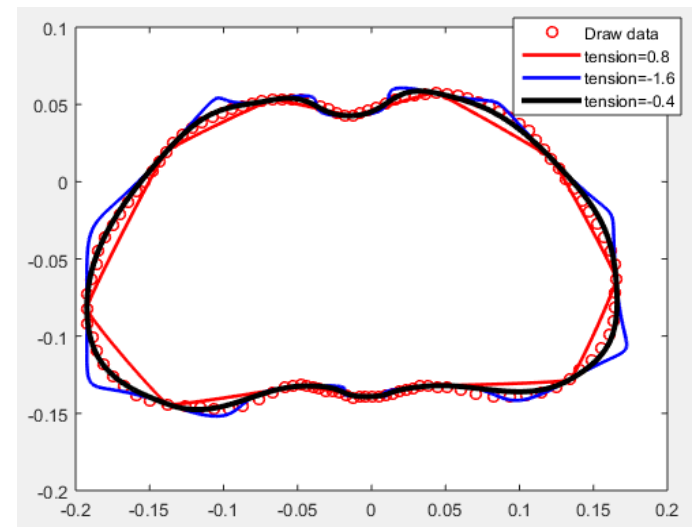

Fig. 13. Interpolation Curves from 12 Key Points with different Tension Parameters.

Algorithm 4 Generating all points (data) from key points by Hermite Curve Interpolation method.

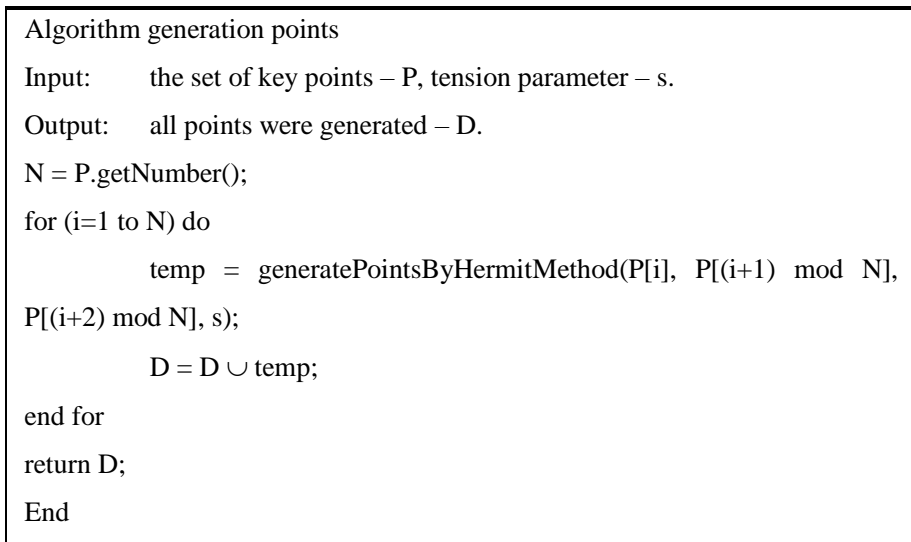

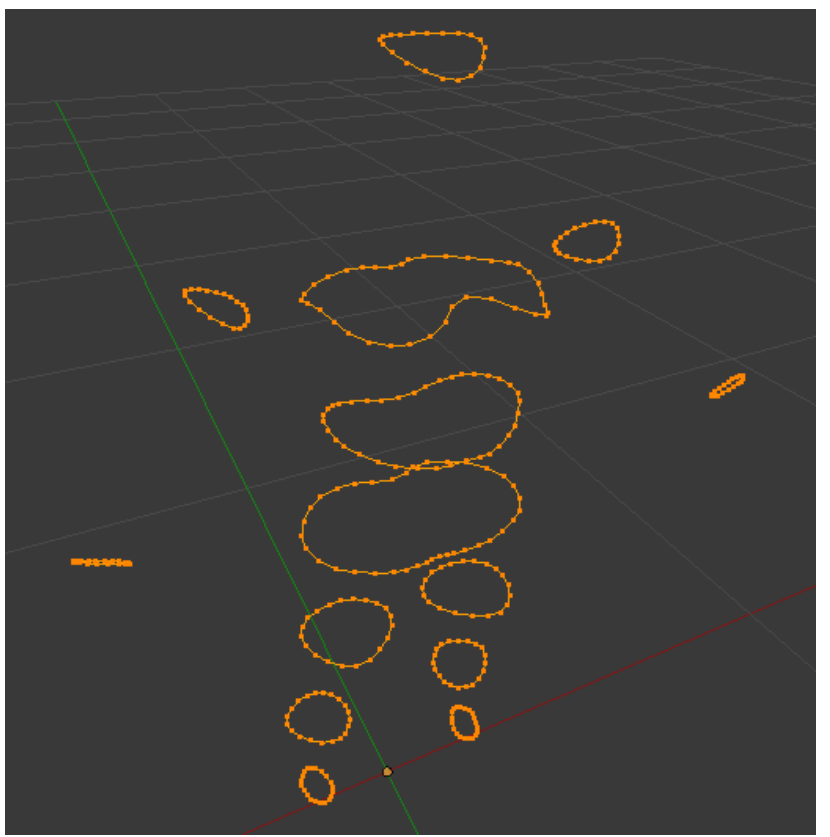

Fig. 14. The Principal Slices on the Body after Interpolation.

7) Building interpolation models from principal slices on parts of the body: Based on the constructed principal slices, we generate extra slices in different parts of the body. The greater the number of slices of each body part, the more detailed it will be, with the tradeoff computational costs. In this research, we chose the number of sections that are sufficient to represent for parts of the body, as shown in Table 5. We use Algorithm 5 to generate these intermediate slices. And result for Algorithm 5 as Figure 15, 16, 17, 18, 19.

TABLE. V. NUMBER OF SLICES FOR EACH REGION

\begin{tabular}{|l|l|l|}
\hline Region & Number of slices & Kind \\
\hline Chest & 6 & 1 \\
\hline Stomach & 10 & 1 \\
\hline Buttocks & 6 & 1 \\
\hline Neck & 9 & 1 \\
\hline Thighs & 14 & 2 \\
\hline Calf & 10 & 2 \\
\hline Leg & 3 & 2 \\
\hline Upper arm & 12 & 2 \\
\hline Lower arm & 8 & 2 \\
\hline Wrist & 5 & 2 \\
\hline
\end{tabular}

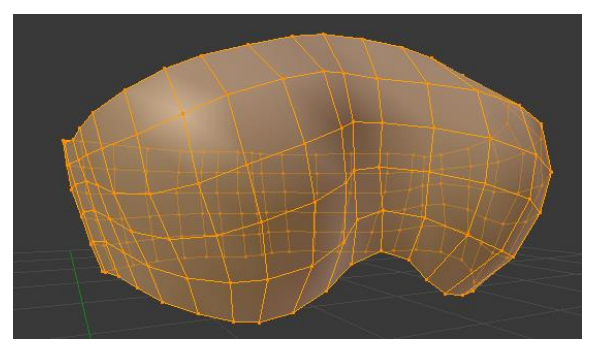

Fig. 15. The slices of the chest region 
Algorithm 5 Generating internal slices of regions.

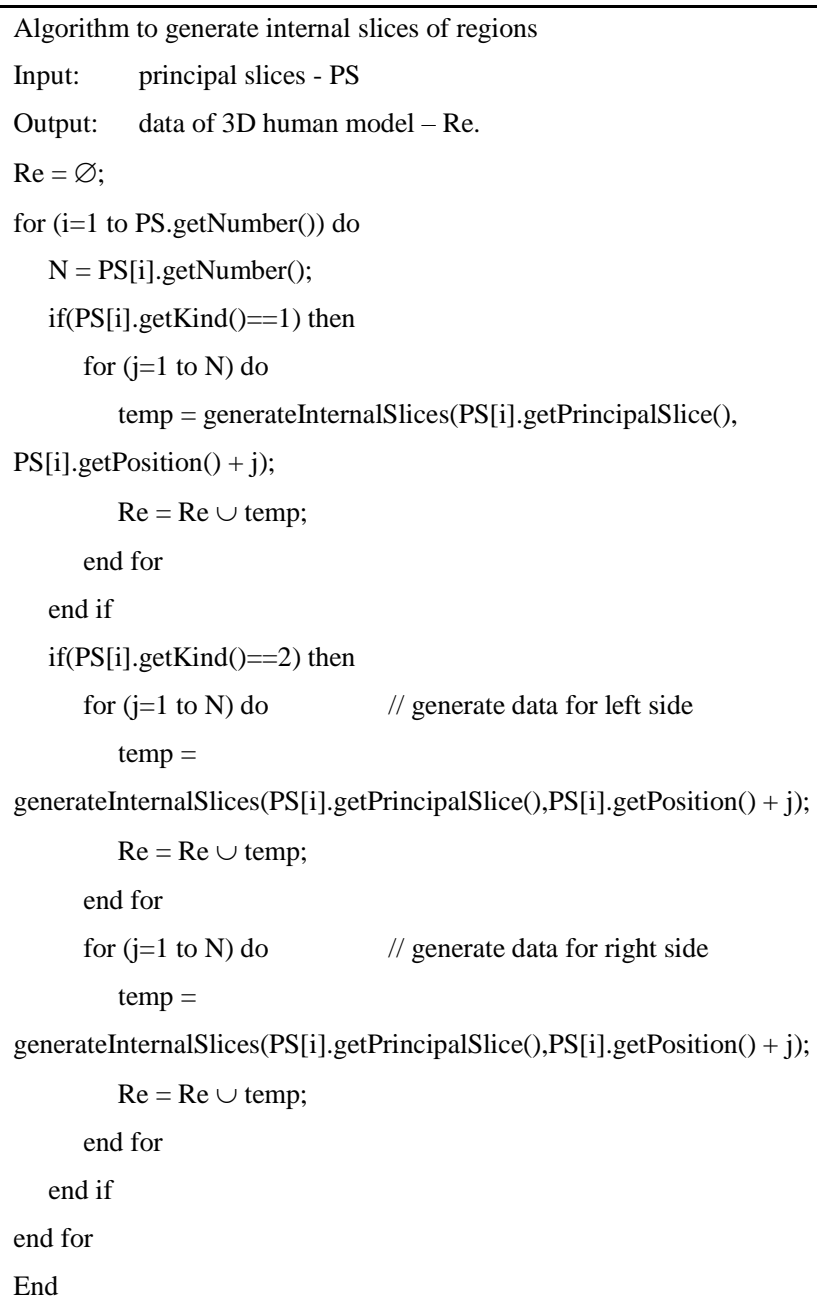

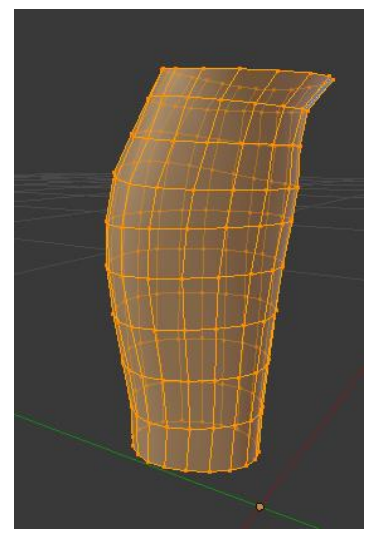

Fig. 18. The Slices of the Calf Region.

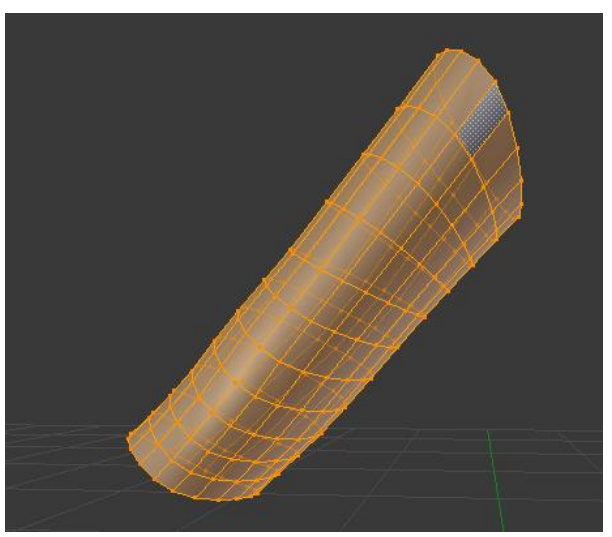

Fig. 19. The Slices of the upper Arm Region.

\section{Error Evaluating Method}

To evaluate errors, we compute the circumference of the slices from the 3D built model. Formula (5) shows how we compute the circumference of slice $\mathrm{i}^{\text {th }}$.

$$
\begin{aligned}
& p_{i}= \\
& \sum_{j=1}^{n-1} \sqrt{\left(x_{i, j}-x_{i, j+1}\right)^{2}+\left(y_{i, j}-y_{i, j+1}\right)^{2}+\left(z_{i, j}-z_{i, j+1}\right)^{2}}+ \\
& \sqrt{\left(x_{i, n}-x_{i, 1}\right)^{2}+\left(y_{i, n}-y_{i, 1}\right)^{2}+\left(z_{i, n}-y_{i, 1}\right)^{2}}
\end{aligned}
$$

where $\mathrm{n}$ is the number of points in slice $\mathrm{i}^{\text {th }}$ and $\left(x_{i, j}, y_{i, j}, z_{i, j}\right)$ are the coordinates of the $\mathrm{j}^{\text {th }}$ poi nt at slice $\mathrm{i}^{\text {th }}$.

$p_{\text {groundtruth }}$ is the circumference of the real data scanned by the 3D scanner, $p_{\text {prediction }}$ is the circumference of slice of the 3D model that we construct from the hand measurements, and $\mathrm{n}$ is the number of models. There are two types of errors that we consider, they are the errors of principal slices and the errors of 3D model.

1) Evaluating the errors of principal slices: The errors of principal slices are the errors that we only calculate at principal slices representing the body's measurements, Formula 6

SizeLoSS $=\frac{\sum_{i=1}^{n}\left(p_{\left.\text {groundruth, } \mathrm{i}-p_{\text {prediction }, \mathrm{i}}\right)^{2}}^{n}\right.}{n}$

Fig. 17. The Slices of the thing Region. 
2) Evaluating the errors of $3 D$ built model: The error of the 3D built model is the error which is computed by slices the whole body of the 3D built model, formula (7).

BodyLoss $=\frac{\sum_{i=1}^{n} \text { BodyLossPerOneModel }}{n}$

with BodyLossPerOneModel $=$ $\frac{\sum_{i=1}^{m}\left(p_{\text {groundtruth }, i}-p_{\text {prediction }, i}\right)^{2}}{m}$

where $\mathrm{m}$ is the number of slices of the whole body.

\section{EXPERIMENTS AND RESULTS}

\section{A. Data and Experimental Environment}

We conduct experiments on the data set of 593 3D scans of women body; each model has a set of manual measurements, and its point cloud is saved under ".obj" format. This dataset was recorded by the garment research group of Hanoi University of Technology [8].

We use Python as the main programming language. The algorithms are implemented on a computer using CPU Intel Core i5, and 4GB RAM. After generating the 3D model, we utilize Blender for visualizing.

\section{B. Results}

1) Program simulating $3 D$ mannequins corresponding to hand measurements: We created a program that simulates the female 3D mannequin corresponding to the manual parameters given by users, as shown in Figure 20. The program helps to observe the actual 3D model and the generated model, Figure 21.

2) Errors: The results of the square error of the principal slices and the squared error of the 3D built model are shown in Table 6 and Table 7.

There were 95 models in the dataset, which were damaged when scanning. These damaged models make the max square error significantly increase, and that leads to the high average error. Figure 22 shows a female 3D model from a defective 3D scanner. Table 8 and Table 9 show the results after eliminating faulty $3 \mathrm{D}$ data.

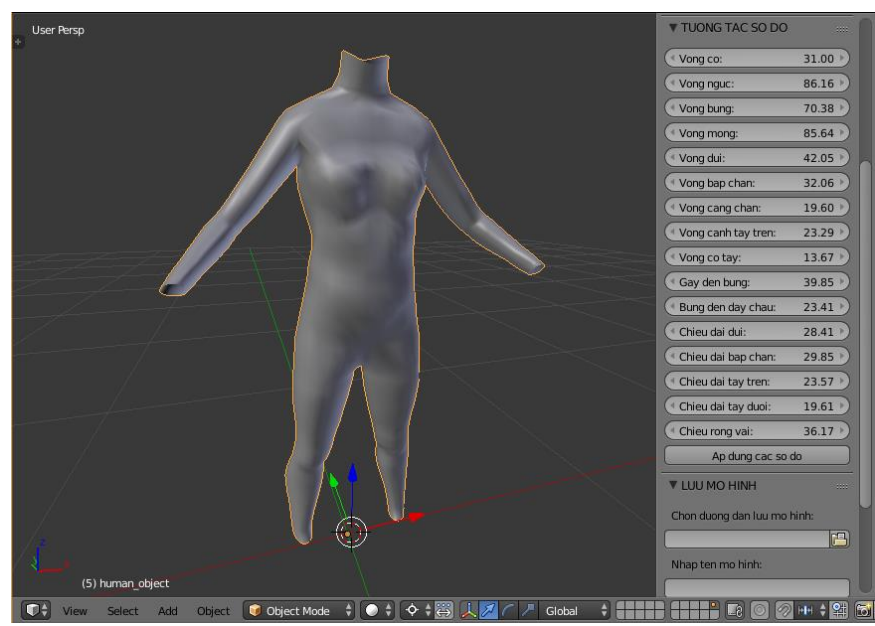

Fig. 20. Simulating 3D Mannequins Corresponding to Hand Measurements.

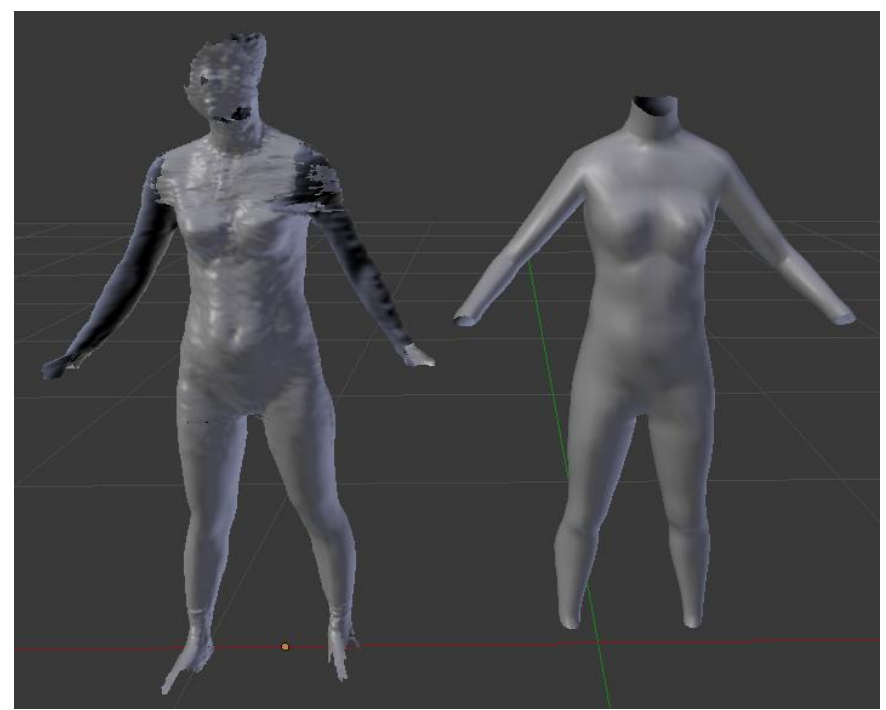

Fig. 21. Female 3D Model: (Left) from 3D Scanner; (Right) Built by Proposed Method.

TABLE. VI. ERRORS OF PRINCIPAL SLICES

\begin{tabular}{|l|l|}
\hline Measuring name & Mean square error \\
\hline Necklace & 2.6129091253 \\
\hline Chest ring & 9.1809139148 \\
\hline Waist circumference & 0.9392992822 \\
\hline Buttocks & 1.0371366978 \\
\hline Middle thigh & 0.0371047278 \\
\hline Calf circumference & 0.0000332125 \\
\hline Leg circumference & 0.0000247387 \\
\hline Upper arm & 1.0321699121 \\
\hline Wrist cuff & 2.0574341568 \\
\hline
\end{tabular}

TABLE. VII. ERRORS OF 3D BUILT MODEL

\begin{tabular}{|l|l|}
\hline Mean square error & 11.02621202 \\
\hline Min square error & 1.38504 \\
\hline Max square error & 125.55837 \\
\hline
\end{tabular}

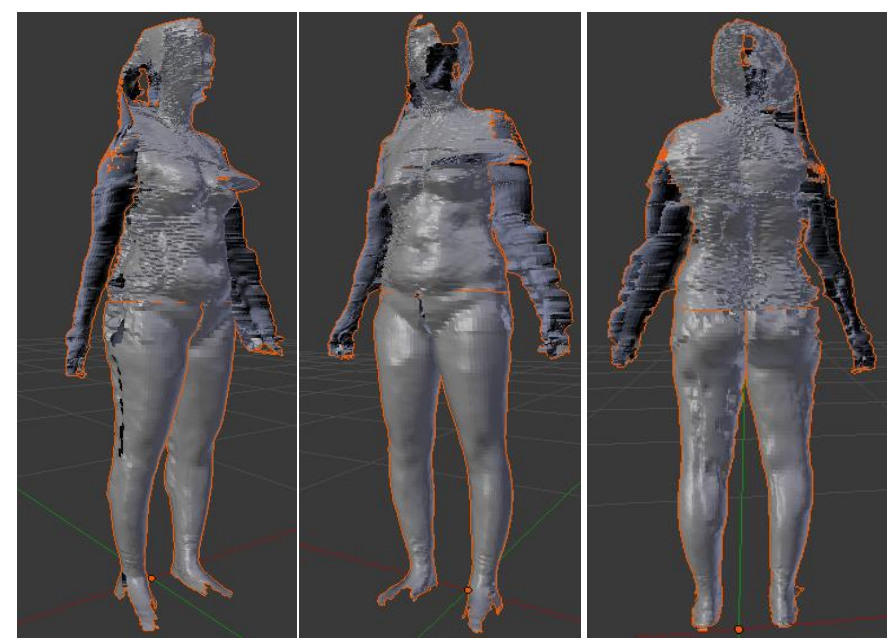

Fig. 22. The Female 3D Model from the 3D Scanner is Wrong. 
TABLE. VIII. ERRORS OF PRINCIPAL SLICES AFTER SEPARATION OF FAULTY DATA

\begin{tabular}{|l|l|l|}
\hline \multirow{2}{*}{ Measuring name } & \multicolumn{2}{|l|}{ Mean square error } \\
\cline { 2 - 3 } & $\mathbf{4 9 8}$ good data & 95 faulty data \\
\hline Necklace & 0.00015151 & 16.3092595715 \\
\hline Chest ring & 0.0001203749 & 57.3076000503 \\
\hline Waist circumference & 0.0000326014 & 5.8630340931 \\
\hline Buttocks & 0.0000614009 & 6.4735945702 \\
\hline Middle thigh & 0.0000339809 & 0.2314334855 \\
\hline Calf circumference & 0.0000394556 & 0.00004396 \\
\hline Leg circumference & 0.0000294007 & 0.0000372449 \\
\hline Upper arm & 0.0000224071 & 6.4427957809 \\
\hline Wrist cuff & 0.0000610313 & 12.8424006462 \\
\hline
\end{tabular}

TABLE. IX. ERrors OF 3D BUILT MODEL AFTER SEPARATION OF FAULTY DATA

\begin{tabular}{|l|l|l|}
\hline & 498 good data & 95 faulty data \\
\hline Mean square error & 5.491083893 & 23.52586951 \\
\hline Min square error & 1.38504 & 10.06982 \\
\hline Max square error & 9.95929 & 125.55837 \\
\hline
\end{tabular}

\section{CONCLUSION}

In this study, a method of building 3D human models is presented. We introduce a framework to decide which primary slices needed to interpolate before generating the entire $3 \mathrm{D}$ model. We conduct the experiments on the dataset of 3D scans of female body and investigate a set of appropriate measurements that used as the input. The proposed method mainly based on the Hermite interpolation, which has a closed form and does not require many calculations, results in a small computational cost.
The main drawback of this approach is that highly depends on the selection of primary slices. Interpolating the intermediate slices only depending on the primary slices of a particular model made a generated model seems reasonable but not practical because of the complexity of the body structure. In practice, However, we rarely have manual measurements at all positions on the human body. One novel way to overcome this drawback is using Deep Learning approach, which takes advantage of information containing in the dataset to infer the intermediate slices.

\section{REFERENCES}

[1] S. Wang, S. Qin and C. Guan, "Feature-Based Human Model for Digital Apparel Design," IEEE Transactions on Automation Science and Engineering, vol. 11, no. 2, pp. 620-626, 2014.

[2] Y. J. Liu, D. L. Zhang, and M. M. F. Yuen, "A survey on CAD methods in 3D garment design," Comput. Ind., vol. 61, pp. 576-593, 2010.

[3] F. Cordier, S. Hyewon, and N. Magnenat-Thalmann, "Made-to-measure technologies for an online clothing store," IEEE Comput. Graph. Appl., vol. 23, pp. 38-48, 2003.

[4] S. Y. Baek and K. Lee, "Parametric human body shape modeling framework for human-centered product design," Comput.-Aided Design, vol. 44, pp. 56-67, 2012.

[5] H. Seo and N. Magnenat-Thalmann, "An example-based approach to human body manipulation," Graph. Models, vol. 66, pp. 1-23, 2004.

[6] Jihong Xu, Wenbin Zhang, "The vacant distance ease relation between body and garment," Second International Conference on Information and Computing Science, Manchester, pp. 38-41, 2009.

[7] Park Soonjee, Miyoshi Machiko, "Development of Theory and Auto CAD Program for Designing the Individual Bodice Pattern from 3D Scanning Data of Human Body", Journal of ARAHE, 10.4, pp.216-225, 2003.

[8] Tran Thi Minh Kieu, Soonjee Park, "Development 'Aodai' pattern for Vietnamese women using 3D scan data", Korean Society for Clothing Industry (KSCI) International Conference, pp.390-391, 2011.

[9] Kochanek D. and Bartels R., "Interpolating Splines with local tension, continuity, and Bias control," Proceedings of the $11^{\text {th }}$ International Conference on Computer Graphics and Interactive Techniques, ACM Press, pp. 33 - 41, 1998. 